\title{
Predictors of new-onset seizures: a 10-year follow-up of head trauma subjects with and without traumatic brain injury
}

\author{
Kalle Vaaramo, ${ }^{1}$ Jussi Puljula, ${ }^{1}$ Sami Tetri, ${ }^{2}$ Seppo Juvela, ${ }^{3}$ Matti Hillbom ${ }^{1}$
}

\begin{abstract}
${ }^{1}$ Department of Neurology, Oulu University Hospital, Oulu, Finland

${ }^{2}$ Department of Neurosurgery, Oulu University Hospital, Oulu, Finland

${ }^{3}$ Department of Clinical Neurosciences, University of Helsinki, Helsinki, Finland
\end{abstract}

\section{Correspondence to}

Dr Sami Tetri, Department of Neurosurgery, Oulu University Hospital, Kajaanintie 52, Oulu 90029, Finland; sami.tetri@ oulu.fi

Received 30 October 2012 Revised 3 May 2013 Accepted 13 May 2013

Published Online First

12 June 2013

\section{Linked}

http://dx.doi.org/10.1136/ jnnp-2013-306267

\footnotetext{
To cite: Vaaramo $K$, Puljula J, Tetri $\mathrm{S}$, et al. I Neurol Neurosurg Psychiatry 2014;85: 598-602.
}

\begin{abstract}
Background It is not known whether alcohol-related head trauma predicts the new-onset seizures, particularly alcohol-related seizures.

Objective We investigated risk factors for new-onset seizures in a cohort of 739 head trauma subjects. Methods All subjects with head trauma attending Oulu University Hospital during 1999, including children and very old people but excluding persons with previous seizures and/or neurological diseases, were enrolled and followed up until the end of 2009. The Finnish National Hospital Discharge Register was used to identify all visits due to seizures during the 10-year follow-up. Dates of death were obtained from the official Cause-of-Death Statistics. Cox proportional hazard regression models and Kaplan-Meier survival curves were used to identify

seizures following TBI, ${ }^{6}$ while another has reported that alcohol abuse may predispose individuals to the occurrence of seizures after cerebral contusion. ${ }^{8}$

Since the risks of subjects with head traumas of differing severity, that is, with or without TBI, developing new-onset seizures, the risk factors involved and the relation of these to alcohol consumption are unknown, we conducted a prospective cohort study among unselected head trauma subjects to demonstrate whether new-onset seizures are associated with alcohol consumption. We hypothesised that an alcohol-related index trauma may be a predictor of new-onset seizures irrespective of trauma severity, as minor head traumas are frequent among those with heavy binge drinking, which in turn may provoke seizures.
\end{abstract} predictors of new-onset seizures.

Results New-onset seizures were observed in 42 out of the 739 subjects (5.7\%). An alcohol-related index injury (adjusted HR 2.50, 95\% Cl 1.30 to $4.82, \mathrm{p}=0.006$ ), moderate-to-severe traumatic brain injury (TBI) as the index trauma $(3.13,1.46$ to $6.71, p=0.003)$ and preceding psychiatric disease $(3.23,1.23$ to 9.21 , $p=0.028$ ) were significant predictors of new-onset seizures during the follow-up after adjustment for age and sex. An alcohol-related index injury was the only independent predictor of the occurrence of an alcoholrelated new-onset seizure (adjusted HR $12.13,95 \% \mathrm{Cl}$ 2.70 to $54.50, p=0.001)$, and these seizures $(n=19)$ developed more frequently among subjects without $(n=14)$ than with $(n=5)$ TBI

Conclusions We conclude that alcohol-related head trauma predicts new-onset seizures, particularly alcoholrelated seizures. A brief intervention is needed in order to prevent the development of alcohol-related seizures.

\section{INTRODUCTION}

Head traumas and acute symptomatic seizures are typical health problems caused by alcohol. ${ }^{1-4}$ Traumatic brain injury (TBI) and substance-use disorders frequently co-occur, ${ }^{5}$ but the relationship between heavy drinking, the severity of a head trauma and seizures is complex. The risk of seizures increases after TBI, but this depends on both the severity and type of injury. ${ }^{6}$ Both moderate-to-severe and penetrating TBI can frequently be followed by seizures, ${ }^{7}$ and one study has found brain contusion with subdural haematoma, skull fracture, loss of consciousness (LOC) or amnesia for more than 1 day and an age of 65 years or older to be significant risk factors for

\section{MATERIAL AND METHODS}

There were 827 subjects, including children and elderly people, admitted to the emergency room (ER) at Oulu University Hospital during 1999 on account of acute head trauma. To investigate the occurrence and predictors of new-onset seizures among these subjects, we excluded from the cohort those who had had a recorded history of seizures, dementia, TBI, brain infarction or other neurological disease before the index trauma. This was done in order to obtain a cohort of subjects with no previous seizure history or any disease known to predispose the carrier to epilepsy. Subjects with a history of psychiatric disease were included, however, because they are prone to drug and/or alcohol abuse. The subjects of this kind in the present series had psychosis $(n=10)$, depression and/or anxiety disorder $(n=9)$ or personality disorder $(n=2)$, and two of them had also received a diagnosis of alcohol dependence before the index trauma. The exclusions left us with a total of 739 subjects who were followed up until the end of 2009.

Acute head traumas were identified in 1999 from the daily checklists kept in the ER. A nurse checked the list and the ER records daily and picked out any notes that suggested a head trauma, including subjects with multiple injuries, for further assessment (by one of the research team). In addition, the hospital discharge register was checked weekly to identify cases of head trauma and TBI that had not been observed on the basis of the ER checklist. This method maximised the possibilities of identifying head trauma subjects. 
The baseline data on all the subjects gathered directly from the hospital charts in 1999 included age at the time of the index trauma, sex, severity of the index head trauma and whether the subject had been under the influence of alcohol at the time of the trauma, together with a history of previous diagnoses. Follow-up data on all the subjects for the period 1999-2009 were then gathered from the National Hospital Discharge (NHD) Register using the record linkage technique, that is, by reference to the subjects' personal social security codes. We did not contact the subjects themselves. The NHD register data included all diagnoses recorded on discharge from any hospital after an inpatient, outpatient or ER visit and also all diagnoses for inpatient visits to health centres (but not for outpatient or ER visits to health centres). Dates of death were obtained from the Statistics Finland Causes-of-Death (SFCD) register. If either the NHD and/or SFCD data included any diagnosis of seizures, we checked the nature of the seizure problem, the possibility of alcohol involvement and other data on the subject in the records of our own hospital as well. A seizure problem was considered to be alcohol-related if it was indicated in the NHD register and/or in our hospital discharge data as being due to acute withdrawal from heavy alcohol drinking. This was possible for those who lived in the catchment area of Oulu University Hospital (89\%).

The severity of the head trauma was divided into three categories according to the presence and severity of TBI: no TBI, mild TBI and moderate-to-severe TBI. The last-mentioned category included all subjects who presented with Glasgow Coma Scale (GCS) $<13$ and also subjects with brain contusion, diffuse axonal injury, traumatic subarachnoid haemorrhage and traumatic intracranial haematomas regardless of their GCS score or need for surgery. Mild TBI included subjects with GCS scores of $13-15$ and LOC $<30$ min or post-traumatic amnesia (PTA) $<1 \mathrm{~h}$ in the absence of traumatic intracranial findings in a brain CT/MRI. ${ }^{9}$ Subjects without a brain CT/MRI were also classified as having TBI if any other criteria for mild TBI were fulfilled. Subjects with a GCS score of 15 and no LOC or no PTA were classified as having head trauma without TBI.

All subjects with alcohol in their blood or breath at the time of sustaining the trauma or were mentioned in the emergency department records as having been found by a nurse or a physician to be under the influence of alcohol were classified as having alcohol-related trauma. ${ }^{10}$ The criteria were a smell of alcohol in the breath combined with signs suggesting alcoholic intoxication, such as unsteady gait, slurred speech or aggressive behaviour. Informed consent is not required in our hospital for the testing of breath or blood alcohol, and samples are usually taken at least from all head trauma subjects with impaired consciousness. In the present series of 739 subjects, breath or blood samples were obtained from 189 (25.6\%), of whom 186 were alcohol-positives, while an additional 83/739 subjects (11.2\%) were judged by professional emergency care providers to be intoxicated.

The data were analysed with IBM SPSS Statistics (V.20). Categorical variables were assessed using conventional statistical methods, including Pearson's $\chi^{2}$ test and Fisher"s exact test, and continuous variables were compared between groups with t-tests. Cumulative rates of developing a seizure were estimated by the Kaplan-Meier product-limit method, and the curves for the groups were compared using the log-rank test. Cox proportional hazard regression models were used to identify independent predictors of a new-onset seizure during the follow-up period, with age, sex, severity of the index head trauma, presence of alcohol in connection with the index trauma and preceding psychiatric disease included in the final predictive model. The proportionality assumption was checked, and the test for significance was based on changes in log (partial) likelihood. A two-tailed $p$ value of less than 0.05 was considered to be statistically significant.

\section{RESULTS}

Baseline data on the 739 subjects are shown in table 1 . These comprised 362 head trauma subjects without TBI, 297 with mild TBI and 80 with moderate-to-severe TBI. Altogether 42 patients developed new-onset seizure(s) during the follow-up period, all attributable to structural or metabolic causes except for one subject who had genetic epilepsy. Alcohol-related seizures were observed in 19 out of the 42 subjects (45.2\%), and were most frequent among those with no TBI. Most subjects developing alcohol-related new-onset seizures (17/19, 89.5\%) had been intoxicated at the time of the index trauma; only one with moderate-to-severe TBI and another without TBI were not.

As expected, the subjects with moderate-to-severe TBI developed new-onset seizures significantly more frequently than others during the follow-up $(p=0.001)$, and mortality during the follow-up was also significantly higher among those with moderate-to-severe TBI initially than in those without TBI (HR $3.64,95 \%$ CI 2.35 to $5.64, \mathrm{p}<0.001)$. On the other hand, those with mild TBI had a lower risk of death than those without TBI $(0.62,0.39$ to $0.99, p=0.047)$. Of the 42 subjects with new-onset seizures, 14 (33\%) died during the follow-up (including 5 with alcohol-related seizures), but the time between the first seizure and death ranged from 0.26 to 9.54 years (median 3.5 years). There was significant heterogeneity in age and sex between the groups $(\mathrm{p}<0.01)$, the subjects with mild

Table 1 Baseline data on the head injury cohort

\begin{tabular}{lcccc}
\hline Variable & No TBI $(\mathbf{n}=\mathbf{3 6 2})$ & Mild TBI $(\mathbf{n}=\mathbf{2 9 7})$ & Moderate-to-severe TBI $(\mathbf{n}=\mathbf{8 0})$ & Total $(\mathbf{n}=\mathbf{7 3 9})$ \\
\hline Age, mean (range)* & $38.0(0-90)$ & $32.1(0-90)$ & $46.1(1-86)$ & $36.5(0-90)$ \\
Sex (men) (\%)** & $271(74.9)$ & $178(59.9)$ & $56(70.0)$ & $505(68.3)$ \\
History of psychiatric disease & $11(3.0)$ & $7(2.4)$ & $3(3.8)$ & $21(2.8)$ \\
Alcohol-related index injury** & $175(48.3)$ & $80(26.9)$ & $17(21.2)$ & $272(36.8)$ \\
New-onset seizure, total* & $22(6.1)$ & $9(3.0)$ & $11(13.8)$ & $42(5.7)$ \\
New-onset seizure, alcohol-related & $14(3.9)$ & $3(1.0)$ & $1(1.5)$ & $7(2.6)$ \\
New TBI before new-onset seizure & $4(1.1)$ & $2(0.7)$ & $33(41.2)$ & $112(15.2)$ \\
Death during follow-up* & $52(14.4)$ & $27(9.1)$ & & \\
\hline
\end{tabular}

${ }^{*} p<0.01$ and ${ }^{* *} p<0.001$ for difference between groups.

TBI, traumatic brain injury. 
Table 2 Risk factors for new-onset seizures among 739 head injury subjects

\begin{tabular}{|c|c|c|}
\hline Variable & $\begin{array}{l}\text { Univariable } \\
\text { HR }(95 \% \mathrm{CI})\end{array}$ & $\begin{array}{l}\text { Multivariable } \\
\text { HR }(95 \% \mathrm{CI})\end{array}$ \\
\hline Age per year & 1.01 (0.99 to 1.02$)$ & 1.00 (0.98 to 1.02$)$ \\
\hline Sex (men) & $1.72(0.82$ to 3.59$)$ & 1.33 (0.62 to 2.83$)$ \\
\hline History of psychiatric disease & $4.05(1.45 \text { to } 11.35)^{* *}$ & $3.23(1.13 \text { to } 9.21)^{*}$ \\
\hline Moderate-to-severe TBI as index trauma & $2.42(1.17 \text { to } 4.98)^{*}$ & $3.13(1.46 \text { to } 6.71)^{* *}$ \\
\hline Alcohol-related index injury & $2.57(1.39 \text { to } 4.76)^{* *}$ & $2.50(1.30 \text { to } 4.82)^{* *}$ \\
\hline
\end{tabular}

TBI being the youngest, followed by those with no TBI. The proportion of men was greatest among the subjects with no TBI and smallest among those with mild TBI, and an alcohol-related index injury was most common among the subjects with no TBI.

Significant and independent risk factors predicting new-onset seizures were identified using the Cox proportional hazard regression model (table 2). After adjusting for age and sex, we found alcohol-related index injury, moderate-to-severe TBI as the index trauma and a preceding psychiatric disease to be significant predictors of a new-onset seizure. We also analysed the subjects separately according to the severity of their head trauma, whereupon the significant independent predictors of a new-onset seizure were a preceding psychiatric disease (HR $4.32,95 \%$ CI 1.24 to $15.12, p=0.022$ ) and an alcohol-related index injury $(3.47,1.26$ to $9.56, \mathrm{p}=0.016)$ among subjects without TBI and an alcohol-related index injury (5.08, 1.07$24.23,0.041)$ and age $(1.04$ per year, 1.01 to $1.08, \mathrm{p}=0.027)$ among the subjects with mild TBI, while none of the parameters was a significant predictor of new-onset seizures among the subjects with moderate-to-severe TBI, perhaps because of the smaller number of subjects. The 21 subjects with a preceding psychiatric disease included four who suffered from new-onset

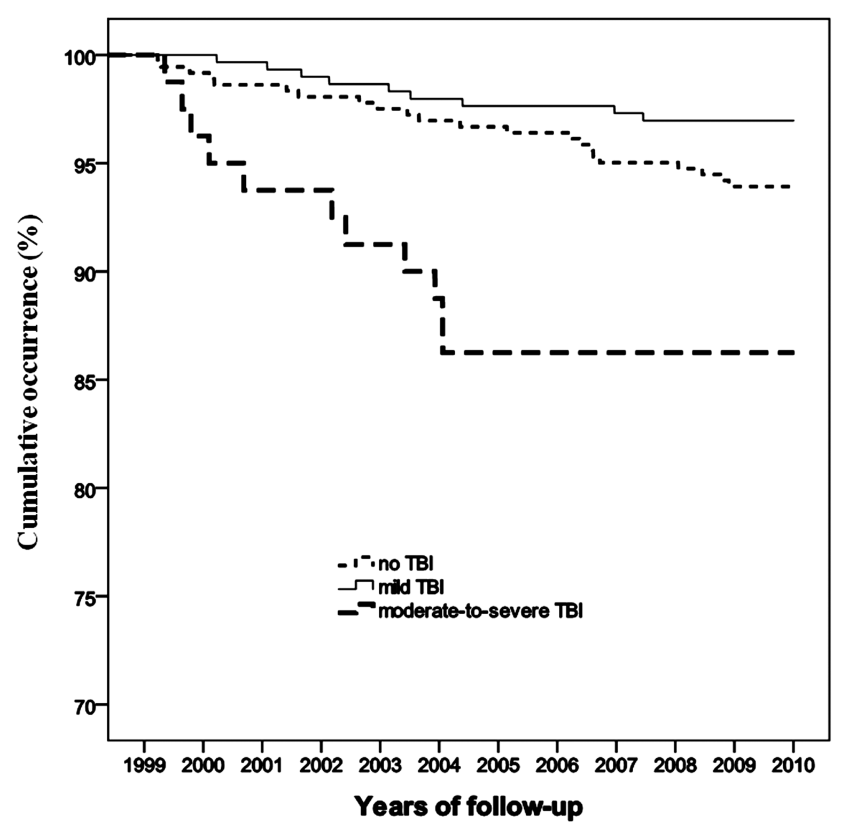

Figure 1 Kaplan-Meier curves showing cumulative rates of new-onset seizures by severity of the head trauma. seizures, two of whom had alcohol withdrawal as one reason for the appearance of a seizure problem.

Cumulative rates of occurrence of new-onset seizures according to severity of the index head trauma are shown in figure 1, where again significant heterogeneity existed between the groups $(\mathrm{p}=0.001)$. New-onset seizures occurred most frequently among the subjects with moderate-to-severe TBI, but they also occurred more frequently among those with no TBI than among those with mild TBI, although the difference between these two groups was not significant. None of those with moderate-to-severe TBI developed a new-onset seizure problem after January 2004.

Cumulative curves for the occurrence of alcohol-related newonset seizures stratified by the presence and absence of TBI in connection with the index trauma are shown in figure 2 . Interestingly, the subjects without TBI developed alcohol-related seizures significantly more frequently $(p=0.029)$. In a separate multivariable Cox analysis adjusted for age, sex, severity of the index head trauma and a history of psychiatric disease, alcoholrelated index injury appeared to be the only independent predictor of the occurrence of an alcohol-related new-onset seizure problem (HR 12.13, 95\% CI 2.70 to $54.50, \mathrm{p}=0.001$ ).

\section{DISCUSSION}

One new finding to emerge here was that, independent of any confounding factors, an alcohol-related head trauma was a significant predictor of new-onset seizures among subjects with no TBI or mild TBI. Furthermore, a history of psychiatric disease was also a significant predictor of new-onset seizures in the total cohort and among those without TBI, a finding that was quite likely explained by concomitant alcohol and/or drug abuse.

Our observation that among $45 \%$ of the subjects with a newonset seizure problem the underlying cause was alcohol abuse is in accordance with previous studies. Alcohol-related seizures are a common form of acute non-genetic seizure, ${ }^{4}$ accounting for about $41-49 \%$ of all ER acute seizure problems. ${ }^{1}{ }^{11}$ Furthermore, seizures are known to be provoked by long-term heavy drinking ${ }^{12}$ and are frequently associated with alcohol dependence. $^{13}$ All acute seizure problems reaching the ER, including alcohol-related seizures, require careful evaluation, because they carry a potential for many serious complications, including acute TBI. ${ }^{1}$ Also, an alcohol-related seizure is an indicator of alcoholism, which frequently leads to premature death. ${ }^{14}$ In the present series, a third of the subjects with newonset seizures died during the follow-up.

Few studies have dealt with the efficacy and effectiveness of brief interventions among subjects with TBI, although many reports have considered this problem among subjects with trauma $^{15}$ and a few with facial trauma. ${ }^{16}{ }^{17}$ It has been shown, however, that a brief intervention may be effective in reducing 


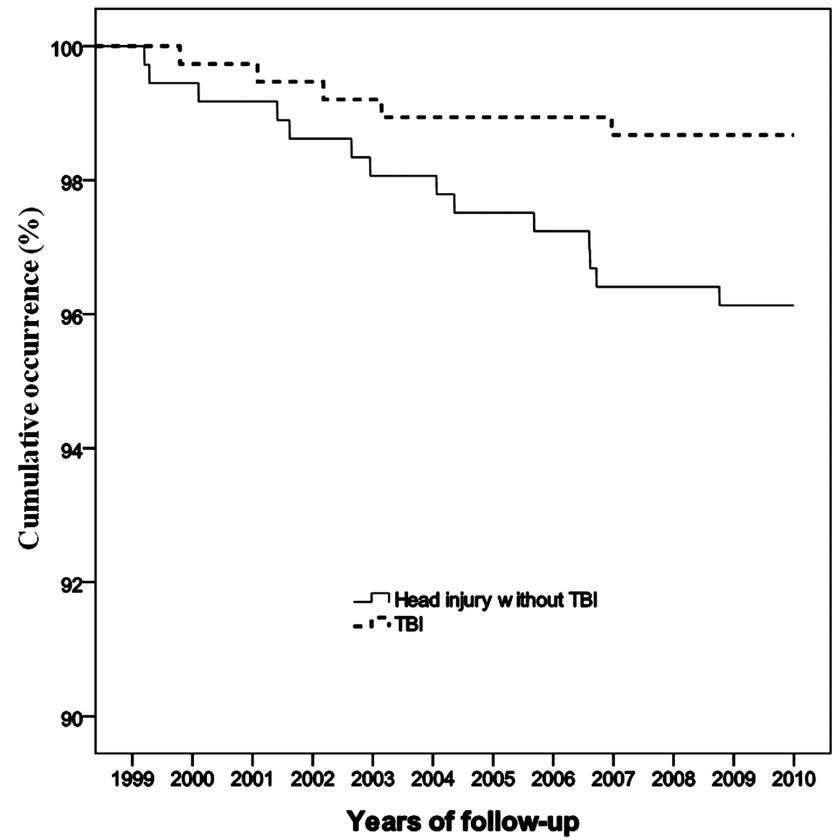

Figure 2 Kaplan-Meier curves showing the cumulative occurrence of alcohol-related new-onset seizures by presence/absence of traumatic brain injury.

alcohol use. ${ }^{15} 18$ The American College of Surgeons requires trauma centres to provide screening and a brief intervention for persons showing a risk of misuse of alcohol or other substances, ${ }^{19}$ and it is feasible to assume that a brief intervention provided for subjects with head trauma would also reduce posttraumatic misuse, especially where the trauma is mild and there is no cognitive deficit. Persons who themselves attribute the head trauma to alcohol misuse may be more open to changing their behaviour in future. ${ }^{20}$ It is often the case, however, that no intervention is performed because of cynicism or a shortage of time.

We observed a preceding psychiatric disease to be a significant predictor of new-onset seizures in our cohort. Psychiatric problems are often associated with drug and/or alcohol abuse, ${ }^{21}$ and this was also the case in our population, but the number of subjects with a preceding psychiatric disease and new-onset seizures was small and our observations should be interpreted with caution. A recent report has observed that new-onset seizures were frequently preceded by psychosis, depression, bipolar disorder and anxiety disorder. ${ }^{22}$ We did not evaluate the role of antipsychotics and/or antidepressants in this study.

It has been shown previously that the risk of seizures depends on the severity of TBI, ${ }^{6}$ and as expected, the risk was high among our subjects with moderate-to-severe TBI. Even subjects with relatively severe TBI may have prior abuse of alcohol and drugs, and these problems should be tackled during rehabilitation. As emphasised by Corrigan, ${ }^{2}$ interventions on account of alcohol and drug problems are largely neglected in cases of subjects with more severe TBI, in spite of the fact that these subjects, too, may suffer from alcohol and drug abuse later on. ${ }^{23}$ To prevent future problems, every head trauma subject should be assessed for alcohol consumption and one should try to influence the drinking habits of intoxicated subjects during their visit to the ER no matter how severe the injury is. This is admittedly difficult, because busy ER physicians tend to discharge all those with a minor trauma as soon as possible.
There are several limitations that should be noted when interpreting our results. First, because the number of subjects with alcohol-related seizures was relatively small, all our observations should be interpreted with caution. For example, a larger number of subjects with moderate-to-severe TBI would be needed in order to show whether an alcohol-related index injury will also predict the onset of alcohol-related seizures in this group. Second, register-based data are prone to bias on account of deficient or inaccurate coding. We tried to reduce this type of bias by checking the data against the available hospital charts. Third, some subjects who develop seizures may not seek help from healthcare providers and may therefore remain unrecorded. Finally, the recording of alcohol abuse prior to a seizure problem may remain unnoticed. The strengths of this work lie in the fact that the data in the Finnish registers (NHD and SFCD) are accurate and the baseline data for 1999 were gathered systematically by means of a structured protocol.

In conclusion, we observed a significant role of alcohol abuse as a predictor of new-onset seizures among subjects with minor head trauma. The need for efficient and effective intervention was apparent. Means for tackling alcohol problems are not usually available to ER personnel in our country.

Contributors KV contributed to conception and design of this study, acquired the data and analysed the data; drafted the manuscript; and approved the final revised version of the manuscript. JP contributed to conception and design of this study and acquired the data; revised the manuscript critically for important intellectual content; and approved the final revised version of the manuscript. ST contributed to conception and design of this study, acquired the data and analysed the data; and revised the manuscript critically for important intellectual content; and approved the final revised version of the manuscript. SJ contributed to conception and design of this study, acquired the data and analysed the data. He is responsible for the accuracy of statistical methods; revised the manuscript critically for important intellectual content; and approved the final revised version of the manuscript. MH contributed to conception and design of this study, acquired the data and analysed the data; drafted and revised the manuscript critically for important intellectual content; and approved the final revised version of the manuscript.

\section{Competing interests None.}

Ethics approval The protocol was approved by the ethical committee of Oulu University Hospital and by the Oulu Provincial Administration.

Provenance and peer review Not commissioned; externally peer reviewed.

\section{REFERENCES}

1 Hillbom ME. Occurrence of cerebral seizures provoked by alcohol abuse. Epilepsia 1980;21:459-66.

2 Corrigan JD. Substance abuse as a mediating factor in outcome from traumatic brain injury. Arch Phys Med Rehabil 1995;76:302-9.

3 Parry-Jones BL, Vaughan FL, Miles Cox W. Traumatic brain injury and substance misuse: a systematic review of prevalence and outcomes research (1994-2004). Neuropsychol Rehabil 2006;16:537-60.

4 Beghi E, Carpio A, Forsgren $\mathrm{L}$, et al. Recommendation for a definition of acute symptomatic seizure. Epilepsia 2010;51:671-5.

5 Olson-Madden JH, Brenner LA, Corrigan JD, et al. Substance use and mild traumatic brain injury risk reduction and prevention: a novel model for treatment. Rehabil Res Pract 2012:2012:174579.

6 Annegers JF, Hauser WA, Coan SP, et al. A population-based study of seizures after traumatic brain injuries. N Engl J Med 1998;338:20-4.

7 Bazarian JJ, Cernak I, Noble-Haeusslein L, et al. Long-term neurologic outcomes after traumatic brain injury. J Head Trauma Rehabil 2009;24:439-51.

8 De Reuck J. Risk factors for late-onset seizures related to cerebral contusions in adults with a moderate traumatic brain injury. Clin Neurol Neurosurg 2011;113:469-71.

9 Vos PE, Alekseenko Y, Battistin L, et al. Mild traumatic brain injury. Eur J Neurol 2012;19:191-8.

10 Puljula J, Savola 0, Tuomivaara V, et al. Weekday distribution of head traumas in patients admitted to the emergency department of a city hospital: effects of age, gender and drinking pattern. Alcohol Alcohol 2007;42:474-9.

11 Earnest MP, Yarnell PR. Seizure admissions to a city hospital: the role of alcohol. Epilepsia 1976:17:387-93.

12 Victor M, Brausch C. The role of abstinence in the genesis of alcoholic epilepsy. Epilepsia 1967:8:1-20. 


\section{Epilepsy}

13 Chan AW. Alcoholism and epilepsy. Epilepsia 1985:26:323-33.

14 Pieninkeroinen IP, Telakivi TM, Hillbom ME. Outcome in subjects with alcohol-provoked seizures. Alcohol Clin Exp Res 1992;16:955-9.

15 Corrigan JD, Bogner J, Hungerford DW, et al. Screening and brief intervention for substance misuse among patients with traumatic brain injury. J Trauma 2010;69:722-6.

16 Smith AJ, Hodgson RJ, Bridgeman K, et al. A randomized controlled trial of a brief intervention after alcohol-related facial injury. Addiction 2003;98:43-52.

17 Shetty V, Murphy DA, Zigler C, et al. Randomized controlled trial of personalized motivational interventions in substance using patients with facial injuries. I Oral Maxillofac Surg 2011;69:2396-411.

18 Blow FC, Barry KL, Walton MA, et al. The efficacy of two brief intervention strategies among injured, at-risk drinkers in the emergency department: impact of tailored messaging and brief advice. J Stud Alcohol 2006;67:568-78.
19 American College of Surgeons. Resources for the optimal care of the injured patient 2006. Chicago, IL: American College of Surgeon, 2006.

20 Sander AM, Bogner J, Nick TG, et al. A randomized controlled trial of brief intervention for problem alcohol use in persons with traumatic brain injury. J Head Trauma Rehabil 2012:27:319-30.

21 Murthy P, Chand P. Treatment of dual diagnosis disorders. Curr Opin Psychiatry 2012:25:194-200.

22 Adelöw C, Andersson T, Ahlbom A, et al. Hospitalization for psychiatric disorders before and after onset of unprovoked seizures/epilepsy. Neurology 2012:78:396-401.

23 Bombardier $\mathrm{CH}$, Temkin NR, Machamer J, et al. The natural history of drinking and alcohol related problems after traumatic brain injury. Arch Phys Med Rehabil 2003;84:185-91. 